\title{
Glaubensgemeinschaften auf Lombok
}

\section{Übersicht}

Lombok, eine der Kleinen Sundainseln, liegt unmittelbar östlich von Bali und ist von Denpasar aus in zwanzigminütigem Flug erreichbar. Kleinere Schiffe laufen von Padang Bay (Ostbali) die Westküste Lomboks an. Die Fläche der Insel beträgt $4600 \mathrm{~km}^{2}$, also um rund $750 \mathrm{~km}^{2}$ weniger als diejenige Balis. Die Insel ist gebirgig. Die nördliche, hohe Kette mit dem Gunung Rinjani $(3775 \mathrm{~m})$ wird durch eine von Westen nach Osten verlaufende große Mulde von der niedern, südlichen Kette (Höhen unter $300 \mathrm{~m}$ ) klar getrennt. $200 \mathrm{~km}$ südlich von Mataram hebt sich als einzige Kuppe der Gunung Maseje (729 m) aus dem Landschaftsbild heraus. Die Südküste vor allem ist außerordentlich stark gegliedert und besitzt gute Anlegeplätze für Schiffe. Geologisch betrachtet, wird die Insel durch die tiefe Straße von Lombok von Bali getrennt. Flora und Fauna der Inseln unterscheiden sich denn auch deutlich voneinander. Der Norden Lomboks erhält während der Nordwest-Monsunzeit (Oktober/November bis März/April) Niederschläge, deren Menge durch den Staueffekt der hohen Vulkankette vergrößert wird. Das Plateau hingegen, das im Lee dieser Barriere liegt, wird auch während der Regenzeit nicht ausgiebig befeuchtet. Der Südostpassat bedingt die Trockenzeit, die hier ausgeprägter ist als auf Bali. Da nur lokal bewässert werden kann, ernten Lomboks Bauern meistens nur einmal im Jahr Reis. Trockene Jahre, wie z. B. 1972, führen zur Verknappung des Hauptnahrungsmittels und zu Preissteigerungen. Im Januar 1973 war deshalb der Reispreis auf Lombok beinahe doppelt so hoch wie derjenige auf Bali.

Die Bevölkerung (700000 Einwohner) gehört verschiedenen Religionsgruppen an, von denen jede ihre Eigenständigkeit stark betont.

\section{Religiöse Gemeinschaften}

Es sind dies: 1) Animisten, 2) "Buddhisten», 3) Muslim, 4) balinesische Hinduisten, 5) Wetu Telu (Wetu Tiga). Von diesen fünf Gruppen werde ich vor allem die Wetu Telu ausführlich besprechen.

\section{1) Die Animisten}

Der Animismus als ursprünglichste Religion findet sich noch in den unzugänglichen Berggebieten der
Insel. Viele animistische Kultformen tauchen in irgend einer Form aber auch in den traditionellen Zeremonien der Wetu Telu, der «Buddhisten» und der balinesischen Minderheit auf Lombok auf. Die Verehrung von Naturerscheinungen und die Steinkulte zählen dazu.

\section{2) "Buddhisten»}

Im Gegensatz zu Bali, wo das buddhistische Gedankengut im hindu-javanisch-balinesischen Glauben aufgegangen ist, hat auf Lombok eine Minderheit von 14000 bis 15000 «Buddhisten» ihre Eigenständigkeit teilweise bewahrt. Ihr Glaube ist freilich nur noch entfernt mit dem ursprünglichen Buddhismus verwandt.

Die Begriffe des Hinayana und Mahayana sind den Buddhisten Lomboks unbekannt. Einige ihrer Grundsätze erinnern jedoch an das Gedankengut des Mahayana. Der nur spärlich vorhandenen Überlieferung nach soll der Buddhismus von Java über Bali und Sumbawa nach Lombok gelangt sein. In drei Siedlungen wurden unter Führung buddhistischer Mönche geschlossene Kampung gegründet, nämlich in:
1) Barusatan (dem heutigen Gangga)
2) Orong Empak Penasan (Tanjung)
3) Celiman Irang (Pemenang)

Da diese Gemeinden von den buddhistischen Kulturzentren vollständig abgeschlossen waren, unterlagen sie vor allem seit Beginn des 17. Jahrhunderts der starken balinesisch-hinduistischen Beeinflussung, die um so wirksamer war, als schon bald keine buddhistischen Mönche mehr zur Führung der Gemeinschaft da waren. Heute leiten balinesisch-hinduistische Pemangku auch die Zeremonien der «Buddhisten». Im Unterschied zum Hinduismus kennen Lomboks «Buddhisten» keine Kastengliederung.

Eigentliche Tempelanlagen fehlen. Wohl sind einige Stupa vorhanden, doch enthalten sie keine Reliquien. Auf dem Stupa-Platz finden gemeinsame Andachten statt. Der Glaube ist einerseits im Kharma Phala verankert, anderseits verehren die «Buddhisten» Lomboks aber auch ihre eigene Gottheit, nämlich Bhatara Cenang (den «Beschützer»). Die

Dr. A. Leemann, Brüschhalde 30, 8708 Männedorf 
Predigten ihres großen Lehrers sind den «Buddhisten» nicht mehr bekannt. Die geistigen Führer der heutigen Lombok-Buddhisten, die Gebrüder Martinom in Tanjung, versuchen, das vollständige Aufgehen der buddhistischen Religion im balinesischhinduistischen Gedankengut dadurch zu verhindern, $\mathrm{da} ß$ in nächster Zukunft javanische Bhikkhu - oft in Bangkok geschult - nach Lombok eingeladen werden sollen. Mit Hilfe der Verbreitung buddhistischer Literatur soll eine Neubesinnung stattfinden. Die meisten Angehörigen der 19 Banjar Buddha sind Bauern; wenige sind Kaufleute.

Als einzige Religionsgruppe kennen die «Buddhisten» den Kecodak (eine Tanzform), den sie gelegentlich bei Festen im Kampung tanzen.

\section{3) Die Muslimen}

Rund die Hälfte der Bewohner Lomboks sind Sunniten. Sie wohnen hauptsächlich im Abschnitt Mataram-Labuhanhadj. In Ostlombok bestehen geschlossene Muslimsiedlungen. Die Verbreitung des Islams in Ostlombok wird vor allem auf den Kontakt mit den Bugi (Celebes) zurückgeführt, während sich in Westlombok der Einfluß javanischer Muslimen auswirkte.

\section{4) Die balinesisch-hinduistische Glaubensgemein- schaft}

Der balinesische Bevölkerungsanteil liegt unter 20 Prozent der Gesamtbevölkerung Lomboks. Die Bildung balinesischer Gemeinschaften war die Folge der Feldzüge der Könige von Karangasem (Ostbali). Der König Datu Bejangi von Südlombok hatte den Herrscher von Karangasem um Hilfe im Kampf gegen seinen Widersacher Datu Selaparang in Nordlombok gebeten. Zusammen mit den balinesischen Truppen unter Führung von Ida Anglurah Ketut Karangasem konnte er das nördliche Königreich erobern.

Die sehr lange währenden Kriegswirren erlaubten vorerst nur wenige Tempelbauten (Pura Gunung Sari, Pura Gunung Pengsong, Pura Suranadi). Nach dem Friedensschluß wurde dann Cakra Negara gegründet, eine heute blühende Siedlung in Westlombok. Berühmt ist das klassische Geschichtswerk aus dieser Zeit: Negara Kertagama - wie ein Königreich regiert wird. Im nachfolgenden Zeitabschnitt wurden zahlreiche balinesische Kultstätten errichtet.
Erwähnt seien der Lingsar, Pura Narmada und Pura Batu Bolong.

Die balinesische Herrschaft führte nicht nur dazu, $\mathrm{da} ß$ die heutige Bevölkerung Lomboks teilweise balinesischen Ursprungs ist, sondern bewirkte auch, $\mathrm{da} B$ balinesisch beeinflußte Mischreligionen entstanden, wie dies im Falle der zu besprechenden Wetu Telu klar zum Ausdruck kommt. Interne Machtstreitigkeiten jedoch schwächten dann die balinesische Herrschaft über Lombok, so daß es den Holländern in Zusammenarbeit mit den Muslim nicht schwer fiel, die balinesische Macht Ende des 19. Jahrhunderts zu brechen und die Königsfamilie ins Exil zu schicken.

Die kulturelle und religiöse Verbundenheit der balinesischen Lombokbevölkerung mit der Mutterinsel Bali kommt beispielsweise im Aufbau des Gadoh (oberstes «Stockwerk» der Lingsar-Tempelanlage) deutlich zum Ausdruck (Fig. 1).

\section{5) Die Wetu Telu}

a) Die Verbreitung der Wetu Telu. Die Wetu Telu kommen - soweit bisher bekannt - ausschließlich auf Lombok vor. Ihr Stammland ist das frühere Königreich Bayan in Nordlombok. Ungefähr $30 \%$ der Inselbevölkerung gehören dieser Glaubensrichtung an.

b) Die Bedeutung des Begriffes Wetu Telu (Wetu Tiga).

wetu $=$ herauskommen

telu (tiga) $=$ drei

Der Begriff Wetu Telu fußt auf der fundamentalen Trinität aller Dinge. Es wird oft fälschlicherweise behauptet, der Begriff Wetu Telu rühre davon her, daß die Gläubigen im Gegensatz zu den Muslim nur drei tägliche Gebetszeiten einhielten. In Wirklichkeit jedoch kennen die Wetu Telu gar keine festgesetzten täglichen Gebetsverpflichtungen, sondern jeder betet nur dann, wenn er das Bedürfnis dazu hat.

Die fundamentale Trinität aller Dinge wird mannigfaltig nachgewiesen, beispielsweise durch

I Die Dreifaltigkeit: Allah - Mohammed - Adam. Allah ist der allein zu verehrende Gott. Mohammed wird als Rasul Allah anerkannt und als Kontaktperson zwischen Gott und den Menschen betrachtet. Seine Stellung als Prophet wird ab- 
gelehnt. Adam ist das Symbol des menschlichen Körpers, d. h. des unbeseelten Menschen.

II Die drei Gestirne: Sonne - Mond - Sterne (matahari - bulan - bintang)

III Die Dreiteilung der Welt: Himmel - Erde - Wasser (langit - bumi - air)

IV Die drei Arten der Fortpflanzung: lebend gebären - Eier legen - vegetative Fortpflanzung (beranak - bertelur - tumbuh)

V Die Dreigliederung des menschlichen Körpers: Kopf - Rumpf - Glieder (kepala - badan - anggota)

VI Die drei Prinzipien menschlichen Aktivseins: schöpferisch tätig sein - fühlen - handeln (cipta - rasa - karya)

c) Glaubensgrundsätze und Rituale der Wetu Telu. Drei Gesetze bestimmen das Leben der Gläubigen:

1) Gedenke nur des einen und einzigen Gottes: Allah. (Satu zat ia itu zat Allah)

2) Erfreue das Herz jedes Menschen. (hormati = Nächstenliebe)

3) Entziehe dich dem Einfluß schlechter Geister. Tue nichts Schlechtes. (mencegah diri dari perbuatan jahat)

Die Wetu Telu zählen sich zu den Muselmanen. Im Sembahyang werden neben den individuellen Gebeten drei besondere Verpflichtungen erwähnt:

1) Sembahyang Jumat. Das Freitagsgebet soll gemeinsam gesprochen werden, damit Allah die Gemeinde segne.

2) Sembahyang Majit. Der Mensch soll gute Werke tun, damit er in seiner Sterbestunde auf möglichst viele gute Taten zurückblicken kann. 3) Sembahyang Idulfitri. Dieses steht im Zusammenhang mit tätiger Nächstenliebe. Es verpflichtet den Wetu Telu zum fitrah (zur Sozialarbeit), das aus Liebe und Wertschätzung des Nächsten getan werden soll. Volkskundlich interessant ist die Tatsache, daß in arabischen Ländern das Fest des Fastenbrechens (aid-al fitr) mit Geschenken an Freunde und Bedienstete, aber auch mit einem besonderen Almosen verbunden ist (zokat alfitr).

Die Nacht vor Idulfitri wird Lailatulqadr (in der Sassaksprache Maleman) genannt. Der 21. des
Ramadan wird als Maleman selikur, der 25. des Ramadan als Maleman selae und der 29. des Ramadan als Maleman siwak likur bezeichnet. In diesen Nächten werden in den Dörfern Rhizinusfackeln angezündet, die wie Tausende von Monden leuchten. In diesen Lichterstunden sollen die heiligen Quransuren vom Himmel auf die Erde herunterkommen. Es wird Wert darauf gelegt, daß das Leben auf dem Kitab Suci al Quran und Sunnah Nabi Kebir Mohammed basiert, allerdings angepaßt an lokale Gegebenheiten. Während des dreitägigen Puasafestes enthalten sich die Wetu Telu vom Essen und Trinken. Sie pflegen in diesen Tagen der Meditation. Als Muslim sind sie zur Rezitierung des Kalimat Sahadat verpflichtet. Zuerst wird dieses Gebet arabisch gesprochen, dann in altjavanischer Sprache. Viele der übrigen zeremoniellen Gebete werden ebenfalls in beiden Sprachen ausgedrückt. Der arabische Urtext des Qurans wird allerdings nur von den Religionsvorstehern verstanden; die übrigen Gläubigen lesen in altjavanischen Lontarbüchern (Qlam Wadi und Jati Swara).

Als Grundsatz für die Gebete gilt das Kebatinan: Das Beten von Herzen. Deshalb sind die Wetu Telu auch nicht zum fünfmaligen täglichen $\mathrm{Ge}$ bet verpflichtet. Beim Beten verharren sie in konzentrierter Meditationsstellung: Die Augen geschlossen, die Ohren taub für jegliche Einflüsse von außen. Alles Weltliche soll vergessen werden. Für alle gelten die Panca Indra: Augen, Ohren, Nase, Mund und Gefühle sollen im Zügel gehalten werden. Durch innere Purifizierung soll der Gläubige rein werden. Er soll kämpfen; nicht gegen seinen Nächsten, wohl aber gegen das Schlechte in seinem eigenen Ich. Die Konzentrationsübung ist unter dem Begriff Neng Ning awas iling bekannt (Neng Ning = Konzentration; awas = trage Sorge; iling = erinnern). Im Gegensatz zum Islam ist den Wetu Telu der Genuß von Schweinefleisch nicht verboten, denn «Alles, was von Gott kommt, ist gut».

Als Versammlungsgebäude dient den Wetu Telu die Mesjid, die mit einem Mihrab ausgestattet ist. Die Gebetsnische liegt im Nordwestteil und ist gegen Mekka gerichtet. Auch die Toten werden so begraben, daß ihr Kopf gegen Mekka 
gerichtet ist. Die Wallfahrt nach Mekka hingegen ist unbekannt. Auch werden die traditionellen Feiertage der Muselmanen nicht eingehalten. Die Gläubigen folgen vielmehr weitgehend dem balinesischen Kalender. Odalan (Tempelgeburtstagsfeier im 210-Tage-Kalender) wird wie bei den Balinesen in Ehren gehalten, hingegen wird Njepi (das balinesische Neujahr) nicht gefeiert. Dabei ist zu beachten, daß der Njepi-Tag auch bei den Balinesen auf Lombok erst in jüngster Zeit bekannt geworden ist. Die Verehrung der vergöttlichten Ahnen erfolgt im Balai Gedé, wo Erinnerungsstücke an die Verstorbenen aufbewahrt bleiben. Pemali sind Orte, an denen Heilige begraben liegen, die hoch in Ehren gehalten werden. Beispielsweise befindet sich ein heiliges Grab der Wetu Telu, zu dem auch balinesische Gläubige ihre Opfer bringen, auf dem Riff von Medane, westlich von Tanjung. Ein Stein, der in einer Vertiefung der Grabplatte liegt, soll demjenigen Glück bringen, der ihn dreimal umdreht und sich nachher die Hand, die den Stein berührte, an die Stirne legt.

d) Geschichtliche Herleitung der Wetu Telu. Nach der mündlichen Überlieferung, die durch schriftliche Quellen bestätigt wurde, ist der Islam unter anderem durch javanische Religionslehrer in Lombok verbreitet worden. Unter den neun Walisanga, die nach der Insel kamen, befanden sich Sunan Kali Jaga (nach der Ethymologie der Wetu Telu abzuleiten von Qodli Zakqa = Lehrer der Weisheit) und Sunan Bunan.

In neuester Zeit versuchen die Wetu Telu ihren Glauben direkt auf das Mutterland des Islam zurückzuführen. Nach den Aussagen von Datoe Soekawati (Mataram) soll Abu Sufijan ${ }^{1)}$ den König von Bayan (Nordlombok) besucht und ihm dabei empfohlen haben, seine Untertanen entweder zu Mohammedanern oder zu Mu'min ${ }^{2)}$ zu machen. Der Herrscher soll sich für den Glauben der Mu'min entschieden haben, von denen die Wetu Telu abgeleitet werden. Mit dieser wohl eher zweckdienlichen als den Tatsachen entsprechenden Erklärung wollen die Wetu Telu heute den sie bedrohenden Sunniten ihre echte Zugehörigkeit zum Islam beweisen. In Wirklichkeit ist der Glaube der Wetu Telu hauptsächlich als Mischung (Agama Kalang) aus Islam und balinesischem Hinduismus zu verstehen. In der geschichtlichen Abfolge wurde das Bekenntnis der Wetu Telu beeinflußt durch 1) den Animismus, 2) den javanischen Buddhismus und Hinduismus, 3) den Islam, 4) den balinesischen Hinduismus (seit den Kriegszügen der Könige von Karangasem). Der Glaube wurde ferner durch die Lokalgeschichte und vor allem durch die lokalen traditionellen Gegebenheiten (Adat) geprägt, wie dies im Kapitel Sitten und Gebräuche beschrieben wird. "Abu Sufijan» schreibt den Kiyai, Lebai, Katib und Mudim das Studium des Quran vor. Jeder Gläubige muß das Kopiagam kennen (Piagam = Dynastie), das die Dynastien der Könige von Bayan, Sokong, Selabarang und Pejangi beschreibt.

e) Sitten und Gebräuche der Wetu Telu. «Lain lubuk lain ikannya, lain padang lain belalangnya» - in verschiedenen Ländern sind auch die Bräuche verschieden.

Die Sitten der Wetu Telu unterscheiden sich tatsächlich sehr von denjenigen des übrigen Indonesien, weisen aber verwandte Züge mit dem balinesischen Brauchtum auf, die besonders deutlich in den Rites de Passage zum Ausdruck kommen.

Nach der traditionellen Überlieferung kann der Begriff Adat-isti-Adat folgendermaßen gedeutet werden: Ein Gesetz, das die Schöpfung regelt $(\mathrm{A}=$ Alif, bedeutet Beginn; isti = regeln; dat = Schöpfung). Die Religion ihrerseits regelt den Kontakt zwischen Mensch und Gott. In Adat und Religion gibt es im Zeremoniell Parallelerscheinungen. Deshalb arbeiten während der Zeremonien die Religionsvorsteher, die Chefs des Adat und die Regierungsvertreter Hand in Hand. Auch in diesen Ämtern kommt die fundamentale Trinität zum Ausdruck. Es gibt drei Religionsvorsteher: 1) Kiyai, 2) Katib, 3) Lebai: drei Adat-Chefs: 1) Mangkubumi, 2) Jautaka, 3) Serune; drei Regierungsvertreter: 1) Pemusungan (Dorfvorsteher), 2) Kelian (Vorsteher des Kampung), 3) Langlang (Dorfhüter). Jeder dieser drei Gruppen ist eine eigene Farbe zugeordnet: 1) Weiß. Symbol für das Heilige; wird von den Religionsvorstehern getragen. 2) Schwarz und 
Abb. 1: Am Pusuk-Paß bei Linggo Mas. Ein Muslim hat in seiner Neurodung einen Sengaran errichtet. Damit zeigt der Bauer an, daß er das bisherige "Niemandsland" fest in seinen Besitz genommen hat und dieses nun nicht mehr zur allgemeinen Nutzung offen steht. Gleichzeitig dient der Sengaran auch als Fruchtbarkeitssymbol.

Abb. 2: Tanjung: Im Tempel Bebengan Lingsar. Der vom Geist Shivas durchdrungene Pendande (Brahmanen-Priester) leitet am Pujewali-Tag die Tempelzeremonie.
Abb. 3: Tanjung: Am Mandak-Tag führt der Pemangku («Holy Man") den Prozessionszug ans Meer. Im Anblick des Vulkans wird der Gott vom Gunung Agung zur Tempelweihe eingeladen.

Abb. 4: Tanjung: Ähnlich wie auf Bali spielen chinesische Münzen (Bolong) eine bedeutende Rolle als rituelles Geld.
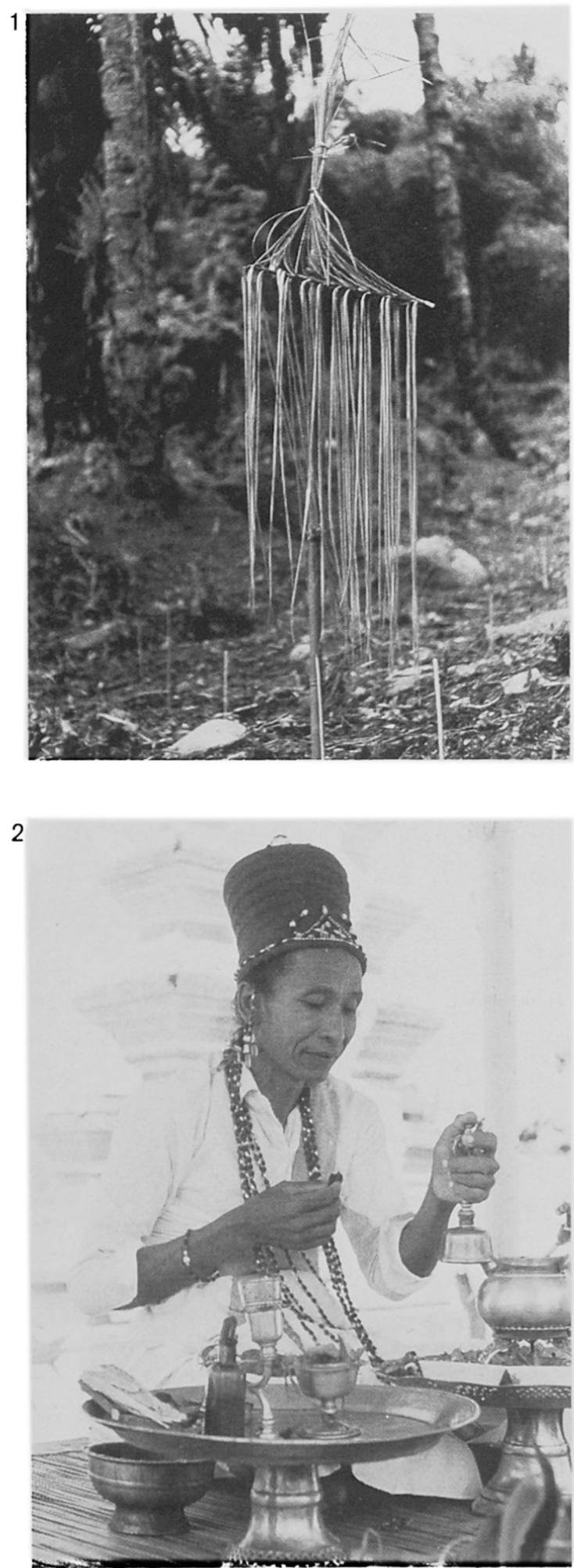
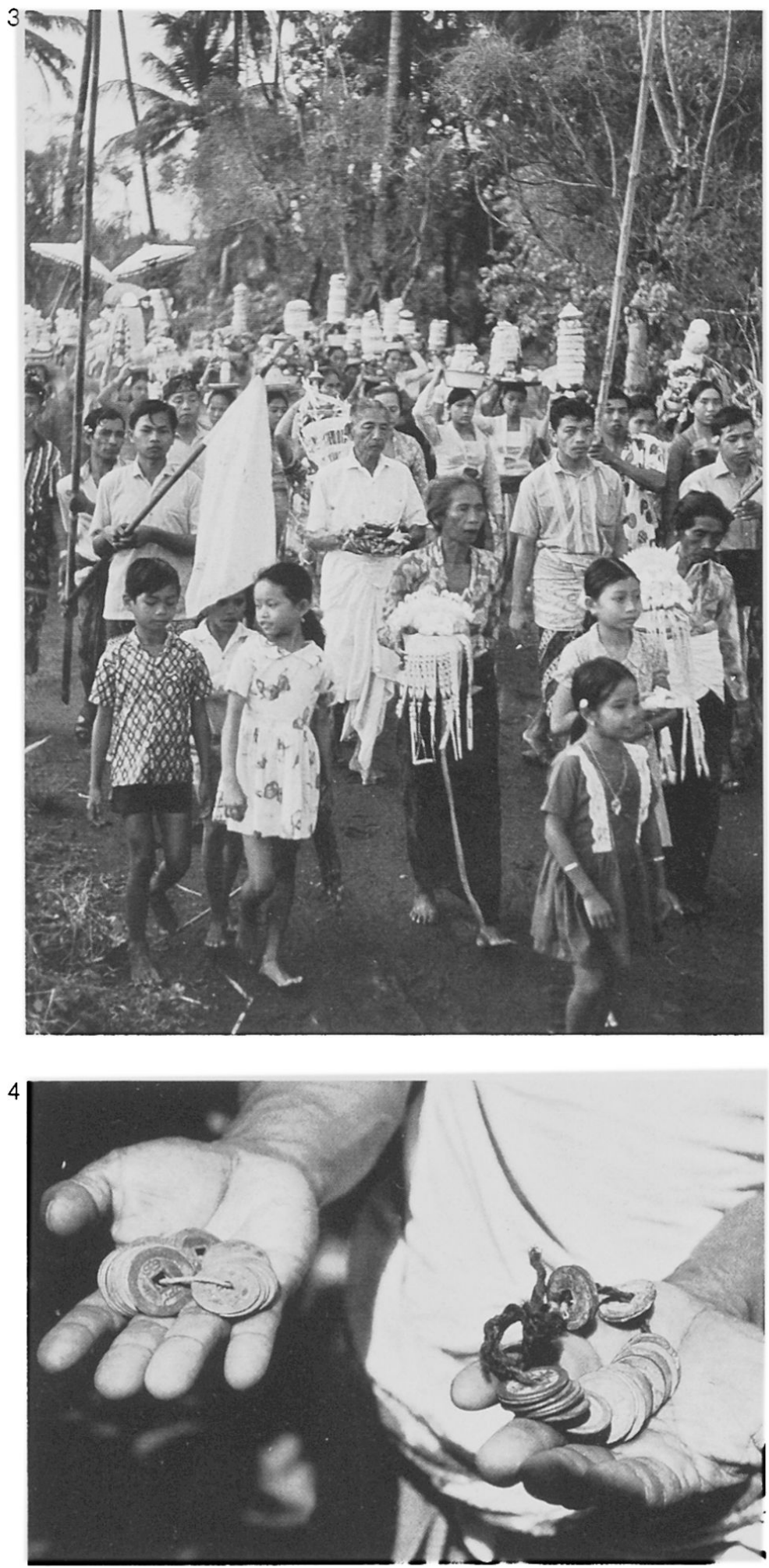
Abb. 5: Tanjung: Im Kampung der Wetu Telu zeigt der Dorfälteste einen Tumbak (zeremonielle Lanze).

Abb. 6: Bayan: Symbolische Tumbakspitzen auf Männergräbern.
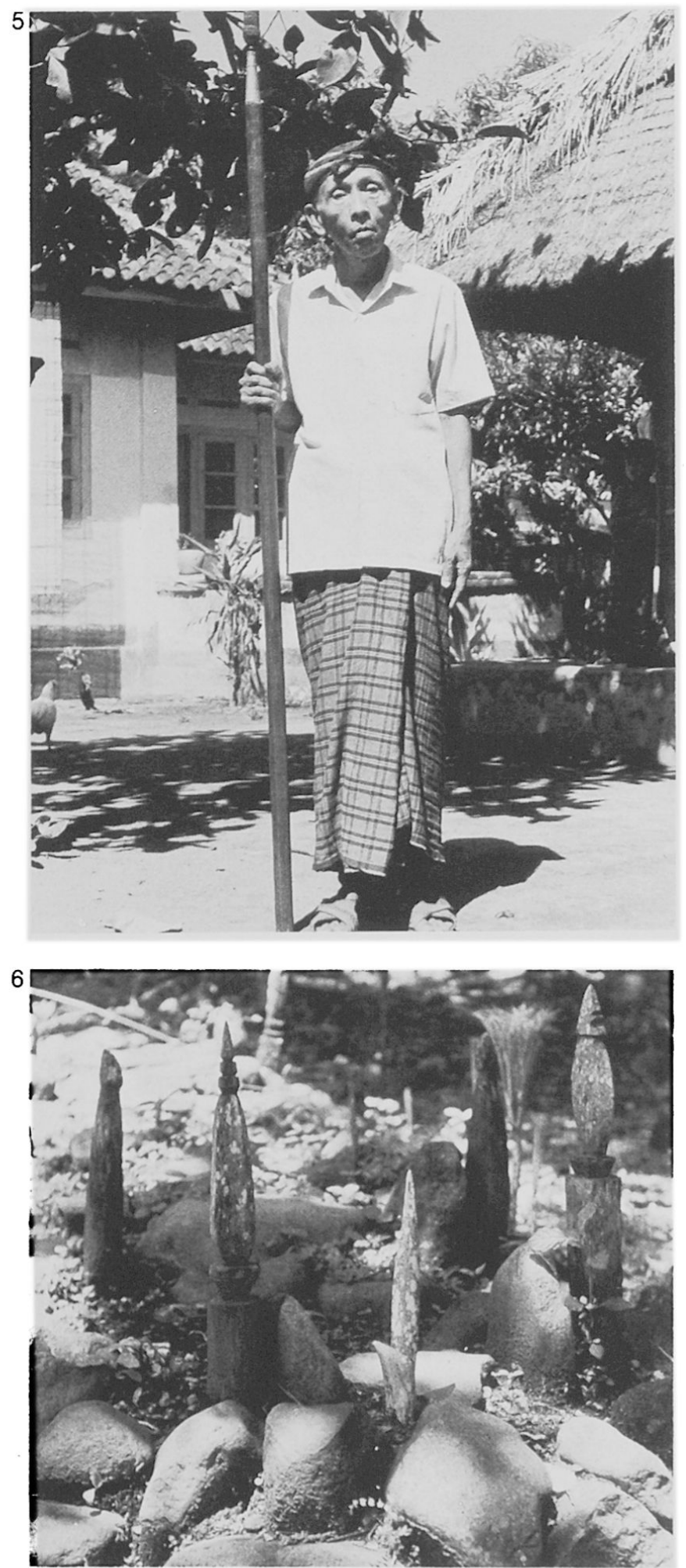

Abb. 7: Tanjung: Datoe Soekawati, einer der geistigen Führer der Wetu Telu, trägt einen Rombong in seinen Händen. Auf der Schale, die Reis und Bolong enthält, befinden sich $1 / 2 \mathrm{~m}$ weißes Tuch, Baumwollfäden und ein Pangot (dolchartiges Messer).

Abb. 8: Bayan: Symbolischer Holzkamm auf einem Frauengrab.
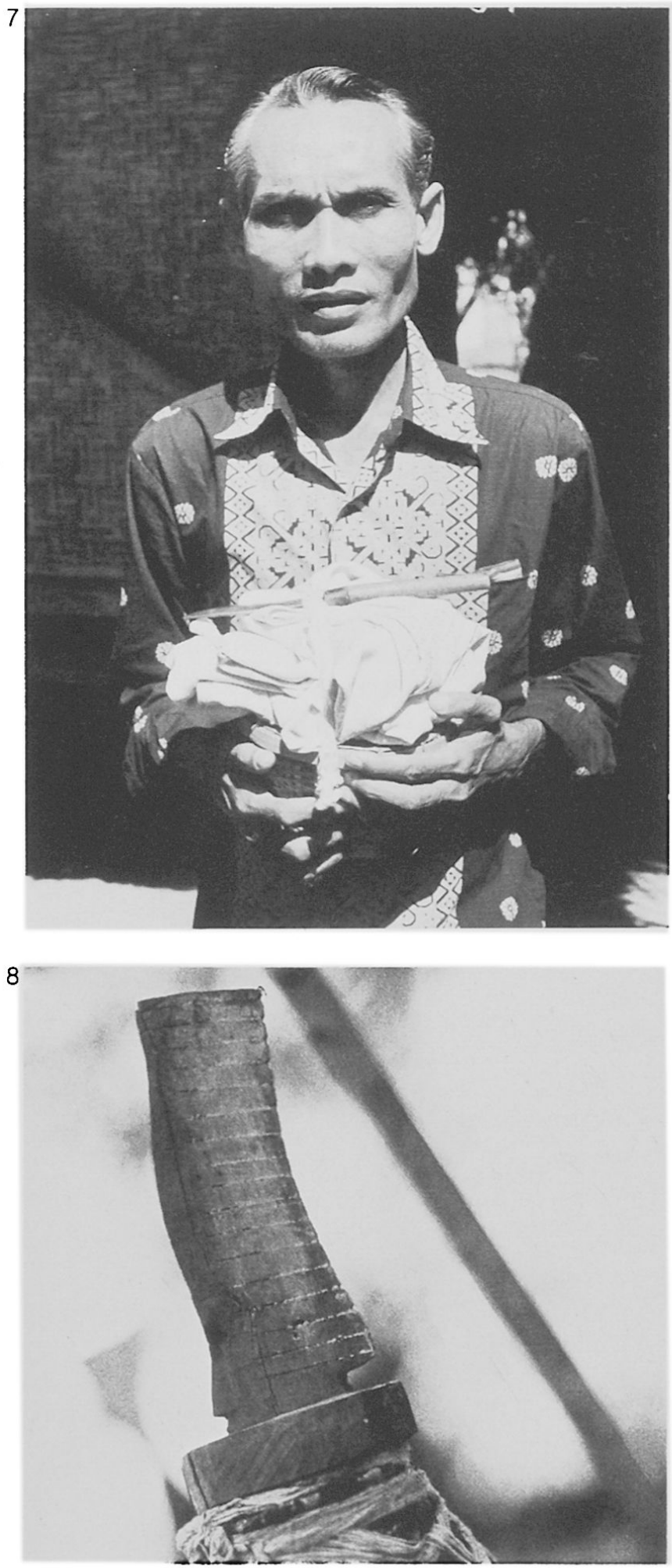
gelb. Symbol für die Abstammung und das Gefühl. Diese beiden Farben kommen den AdatOffiziellen zu. 3) Rot. Symbol für Mut und Ehrlichkeit. Rot wird von den Regierungsvertretern gebraucht.

Die Rites de Passage der Wetu Telu:

1) Adi Kaka: Plazentabeisetzung (adi = klein, kaka $=$ Bruder; in Bali Nanem Ari Ari genannt). Vier Geschwister verlassen zusammen mit dem Neugeborenen den Mutterleib: Blut, Fruchtwasser, Fruchthülle und Plazenta. Wo immer das Kind auch sein mag, werden es seine «vier Geschwister» beschützen. Als Zeichen, daß der Mensch immer mit seinem Geburtsort verbunden bleibt, wird die Plazenta - Vertreter aller vier Geschwister - beigesetzt, und zwar nach der Geburt eines Mädchens links, nach derjenigen eines Knaben rechts vom Hauseingang.

2) Buang Au (Aon); wörtlich übersetzt «Asche streuen». Dieser Ritus kann als Nabelzeremonie bezeichnet werden und ist vergleichbar mit dem Kapus Pungsed in Bali. Der Priester gibt bei dieser Gelegenheit dem Kleinkind den von den Eltern gewünschten Namen.

3) Ngurisang: Dem Kleinkind werden nach drei Monaten (zu 35 Tagen) erstmals die Haare geschnitten. In Bali wird diese Zeremonie Nyambutin genannt.

4) Nyunatang: Wohl nirgends kommt der Mischcharakter der Wetu Telu-Religion deutlicher zum Ausdruck als bei der Nyunatang-Feier. Zwischen 12 und 15 Jahren wird den Knaben die Vorhaut beschnitten, und den Mädchen (manchmal auch den Knaben) werden die Zähne gefeilt. In Bali ist das Zahnfeilen unter der Bezeichnung Metatah bekannt. Zähne sind im Gedankengut der Balinesen ein Ausdruck der Begierde. Domestizierte Tiere, z. B. die Rinder, haben Flachzähne, Raubtiere hingegen - Symbole wilder Kraft besitzen Reißzähne. Ein willensstarker und aktiver Mensch braucht Geist und Kraft. Damit das dualistische Prinzip gewährleistet bleibt, feilt man nur die oberen Schneidezähne und Eckzähne, während die unteren im natürlichen $\mathrm{Zu}$ stand belassen werden.
5) Drei Arten der Heirat:

a) Memadik oder Melamar (in Bali Nganten): abgesprochene Heirat

b) Menyapok (in Bali Mebuncing): Heirat zwischen Cousin und Cousine.

Das Zeremoniell im Falle des Memadik und Menyapok ist einfach und klar. Vor der Heirat wird mit den Eltern des Mädchens Fühlung aufgenommen (Sejati). Im anschließenden Selabar/ Peradang werden die Erbrechte des Mädchens geregelt. Diese Zusammenkünfte werden auch von den Religions-, Adat- und Regierungsoffiziellen besucht; sie regeln die Heirat. Sorong serah ist der Hochzeitstag. Alle Familienangehörigen und die oben erwähnten Offiziellen nehmen an der Zeremonie teil.

c) Memulang (in Bali Ngororod oder Merangkat). Das Mädchen wird den Eltern gestohlen und an einen geheimen Ort entführt. Da sich der Jüngling durch die Entführung des Diebstahls schuldig gemacht hat, muß er bestraft werden. Der Dorfvorsteher als Regierungsoffizieller verabreicht ihm 44 Schläge mit einer Rattanrute. Der Adatvorsteher und der Religionsführer sind dann dafür besorgt und verantwortlich, daß die Hochzeit, von der die Brauteltern nichts wissen, nach den Regeln der Tradition und der Religion durchgeführt wird. Nachher senden die Eltern des Bräutigams einen Botschafter zur Familie der Frau. Ihre Eltern lassen ihm eine schwarze Baumwollschnur (=Basta) zukommen. Bevor der Bräutigam diese erhalten hat, darf er sein Versteck nicht verlassen. Sobald er die Schnur besitzt, muß er sich die Basta um das rechte Handgelenk binden, damit jedermann um den Diebstahl und die Heirat weiß. Dié Großfamilie der Braut setzt nun den Preis fest, den der Ehemann dem Dorfadat gemäß den Brauteltern zu zahlen hat. Die zu entrichtende Summe ist kastenabhängig.

Fall A: Ein Jüngling der ersten Kaste stiehlt ein Mädchen desselben Ranges. Der Tanjung-Adat fordert dafür folgenden Preis:

1) 10000 chinesische Münzen (= Bolong). Umgerechneter Wert: 20 Bolong $=1$ Ringgit $=$ 2,5 Rupien; 1 US $\$=412$ Rupien.

2) 3 Tumbak (zeremonielle Lanzen mit vergoldetem Schaft) 
3) 1 Rombong: Eine Schale, Reis und 225 Bolong enthaltend. Auf der Reisschale: $1 / 2 \mathrm{~m}$ Tuch und 1 Pangot (kleines Messer)

4) 2 junge Kokosnüsse und roten Enauzucker. Fall B: Ein Jüngling der 2. Kaste stiehlt ein Mädchen der 1. Kaste. Das kostet gemäß TanjungAdat:

1) 50000 Bolong

2) 4 Tumbak

3) +4) wie im Fall A

Fall C: Ein Jüngling der 3. oder 4. Kaste stiehlt ein Mädchen der 1. Kaste. Er hat zu zahlen:

1) 100000 Bolong

2) 7 Tumbak

3) +4) wie im Fall A

Die vier Gaben heißen Dasa. Jede hat ihre eigene symbolische Bedeutung. Den Rombong mit Reis erhält die Brautmutter. Mit den 225 chinesischen Münzen wird sie symbolisch für die Muttermilch entschädigt, mit der sie das Kleinkind ernährte. Der Pangot, der unter keinen Umständen je verkauft werden darf, ist für den Vater der Braut bestimmt. Die junge Kokosnuß ist weiß, der Enauzucker rot; sie symbolisieren Knabe und Mädchen. Kokosnuß und Zucker werden von den Familien des Brautpaares gemeinsam gegessen. Damit wird die neue Verbindung bestätigt. Einen Teil der chinesischen Münzen geben die Brauteltern dem Spender wieder zurück, der sie aber nie veräußern darf. Sie müssen in der Familie bleiben.

Erst wenn all dies geregelt worden ist, darf der junge Ehemann die schwarze Schnur an seinem rechten Handgelenk zerschneiden. Die Heirat ist nun legalisiert.

Am 21. 12. 1972 fand in Tanjung eine Wetu TeluHochzeit nach dem Adat von Bayan statt. Vor dem eigentlichen Hochzeitszug marschierten Knaben mit den zeremoniellen Tumbak. Dann folgte das Orchester: Gong, Trommeln und $\mathrm{Zi}$ nellen. Die Frauen waren den begleitenden Männern gegenüber in der Mehrzahl. Auf einer von Jünglingen getragenen Sänfte saß die kunstvoll zurechtgemachte fünfzehnjährige Braut. Im Haar steckte Schmuck aus purem Gold. Als Kleid trug das Mädchen einen silber- und golddurchwirkten Brokatsarong. Auf einer zweiten Sänfte wurde hinter der Braut ihr zwanzigjähriger Gatte getragen; auch er in einem prachtvollen Sarong und mit einem silberdurchwirkten Destar. Vor dem Hause der Brautfamilie nahmen Frauen den Hochzeitszug in Empfang und umtanzten das junge Paar. Die Braut weinte. In einem Aufenthaltsraum im Kampung wurden die Neuvermählten mit dem Rücken zur Wand gesetzt. Dem Manne wurde die Kopfbedeckung abgenommen, und man wusch ihm die Haare mit Seife. Frauen rieben anschließend den nackten Oberkörper des Jünglings und die Gesichter der beiden mit gelber Farbe aus zerriebenen Blumen ein. Später beteiligten sich auch junge Mädchen an dieser Prozedur. Nach der Einfärbung teilte sich der Hochzeitszug. Ein Teil der Gäste begleitete die junge Braut, ein anderer ihren Mann zum öffentlichen Badeplatz. Frauen halfen den beiden bei der Reinigung. Nachher kehrten alle in den Kampung zurück, wo eine Frau der Neuvermählten Reiskuchen reichte. Diese $a ß$ ihn aber nicht, sondern gab ihn, wie es die Tradition vorschreibt, ihrem Gatten weiter.

\section{6) Bestattungsriten:}

1. Zeremonie: Der Verstorbene wird in Abwesenheit des Religionsvorstehers von seinen Angehörigen gewaschen. Mandi Bola = erstes Bad. 2. Zeremonie: Der Tote wird ein zweites Mal, diesmal vom Kiyai oder Penghulu gebadet. Mandi Suci $=$ heiliges Bad.

3. Zeremonie: Der Verstorbene wird in weiße Tücher gehüllt. Auf den Kopf kommt ein weißer Destar. Dann wickelt man den Toten in eine zweite, sackähnliche Hülle.

4. Zeremonie: Der eingehüllte Tote wird auf eine Bambusbahre (= Barang Kurung) gelegt. Im Hause des Dahingeschiedenen rezitiert der Kiyai Quransuren und betet zu Allah. Die Angehörigen verharren vorerst in Meditationsstellung und beten dann zu Gott und zu ihren Ahnen.

5. Zeremonie: Leichenzug zum Friedhof $=$ Nysur Tanah. Das Ausschaufeln des Grabes wird mit dem Buka Gumi Ritual (= Öffnen des Grabes) eingeleitet. Der Kiyai spricht dabei Quransuren in der Sanskritsprache. Der Verstorbene wird so ins Grab gelegt, daß sein Kopf nach Nordwesten (Mekka) gerichtet ist. Während des Talkin-Rituals (talkin $=$ zudecken) rezitiert der Kiyai wei- 
tere Auszüge aus dem Quran, diesmal aber in arabischer Sprache (Selamatan Njusurtanah).

Die Opfergaben der Trauerfamilien werden zunächst auf einem provisorischen Bambusgerüst aufbewahrt und nachher zum Versammlungshaus des Kampung gebracht. Die Gaben bestehen aus Dingen, die im Alltag gebraucht werden, wie Stoffe, Kleider, Kämme, Nahrungsmittel, Petrollampen, Seife, Zigaretten, Zündhölzer usw. Der Kiyai nimmt alle Geschenke entgegen und verteilt sie dann an arme Leute (= Sedekah).

6. Zeremonie: Drei Tage nach dem Tode findet die Nelung-Feier statt (telu $=3$ ). Der Kiyai rezitiert Quransuren.

7. Zeremonie: Ähnlich wie die Dreitagezeremonie gestaltet sich die Siebentagefeier $=$ Mituk (pituk =7).

8. Zeremonie: Vierzig Tage nach dem Ableben wird das Petang puluh (= vierzig)-Ritual durchgeführt, das ähnlich ist wie Zeremonie 6 und 7. 9. Zeremonie: Nyatus Hari (seratus $=100$ ). Hundert Tage nach dem Tode wird ein weiteres Mal des Verstorbenen gedacht.

10. Zeremonie: Tausend Tage nach dem Ableben findet das Nyiu (auch Nyayang genannt) statt. Der Begriff wird von siu = tausend abgeleitet. Dieses Nyiu ist die End- und Hauptzeremonie, die zum Gedächtnis des Dahingeschiedenen gehalten wird.

Am Lebaran-Tag wird das Grab mit Heiligem Wasser (Air Kuluh) geweiht. In Bayan zieren vom Tage der Bestattung an bis zur Tausendtagefeier Holzfiguren die Reihengräber. Die Gräber männlicher Verstorbener haben symbolische Tumbakspitzen, diejenigen der Frauen große hölzerne Haarkämme. Als zusätzlicher Grabschmuck werden Geweihe von Rehböcken verwendet. Während der Tausendtage-Zeremonie entfernt man die Holzsymbole und ersetzt sie durch Steingaben.

Das Grab des Heiligen von Bayan befindet sich in einer verschlossenen Hütte. Wohl garantiert die indonesische Verfassung beim Einhalten gewisser Prinzipien die Religionsfreiheit. Trotzdem haben extreme Muslim mehrmals die Kultstätten der Wetu Telu überfallen und zerstört. Im Spätsommer 1972 richteten deshalb die gei- stigen Führer der Wetu Telu ein Schreiben an das Polizeikommissariat in Mataram. Sie gaben darin dem dringenden Wunsche Ausdruck, die Regierung in Jakarta möge sich ihres Problemes annehmen und Maßnahmen ergreifen, damit die Religionsfreiheit der Wetu Telu auch in der Praxis gewährleistet sei.

7) Andere Riten:

Am Meroah-Tag wird darum gebetet, daß Gott der Menschenseele Gelegenheit gebe, mit ihm in Kontakt zu treten. Während der Puasa-Zeremonie erinnert sich der Gläubige der Sinne und Begierden, die ihn anfechten (upa, wasa $=$ die Wiege unserer Sinne). Dieses Gedächtnis ist mit dreitägigem Fasten verbunden. Am LebaranTag wird der Sieg über die inneren Feinde gefeiert (lebar $=$ siegen).

Ins religiöse Zeremoniell aufgenommen sind auch folgende Speiserituale:

1) Asyra: Die Zubereitung von weißem Porridge

2) Lapar: Die Zubereitung von rotem Porridge

3) Maulud: Das Kochen von gelbem Reis am Geburtstag von Nabi Mohammed

Weiß symbolisiert in diesem Falle den Leim, rot das Blut und gelb den Geschmacksinn. Die Mischung dieser drei Komponenten macht den Menschen aus, der von Gott mit Verstand versehen wurde.

Eine andere Farbdeutung weist auf die vier Elemente hin:

$$
\begin{array}{ll}
\text { Feuer (api) = weiß } & \text { Luft (angen) }=\text { blau } \\
\text { Wasser (air) = gelb } & \text { Erde (tanah) }=\text { rot }
\end{array}
$$

Die Tücher um die Steingaben der Wetu Telu im Lingsar zum Beispiel sind gelb. Sie werden sehr wahrscheinlich dem heiligen Teich gewidmet, an den sich die Gläubigen begeben, um dort um Regen für die Ernte zu bitten.

f) Das Kastensystem der Wetu Telu. Ein ganz wesentlicher Unterschied zwischen dem Glauben der Sunniten und demjenigen der Wetu Telu besteht darin, daß die Gesellschaftsordnung der letzteren auf dem Kastensystem aufgebaut ist.

1. Kaste: Datoe (Sprache: Halus)

2. Kaste: Raden (Sprache: Halus)

3. Kaste: Buling (Sprache: Madia)

Kastenlose: Jajar Karang (Sprache: Kasar) 
Nach der traditionellen Überlieferung müßte man annehmen, daß die neun Walis das Kastensystem vom Königreich Demak auf Java nach Lombok gebracht haben. Der Tatsache entspricht aber wohl eher, daß die von Bali kommenden Eroberer sowohl die Religion als auch die soziale Struktur der unterworfenen Bevölkerung beeinflußten. So heißen zum Beispiel die Kastensprachen gleich wie auf Bali, obwohl die Bevölkerung Lomboks eine andere Sprache, nämlich Sasak, spricht. Auch die vier Kasten der Wetu Telu entsprechen genau denjenigen Balis:
1. Kaste: Brahmanen
3. Kaste: Wesya
2. Kaste: Ksatrya
Kastenlose: Sudra

Nachdem über die Insel Bali bereits eine reichhaltige völkerkundliche Literatur besteht, wäre dringend $\mathrm{zu}$ wünschen, daß sich die Ethnographen in vermehrtem Maße den verschiedenen Bevölkerungsgruppen Lomboks zuwenden würden. Die Gefahr ist groß, daß durch die Erschließung der Insel die Sitten und Gebräuche rasch verflachen.

\section{Literatur}

PAREJA FELIX M.: Islamología (Madrid, 1952-1954)

DATOE POETRAWA: Originalschreiben an Komandas

Korein 162 «Wirabhakti», Mataram (1972)

RONART S. und N.: Lexikon der Arabischen Welt

(Zürich, 1972)

SPEED F. W.: Indonesia today (Singapore, 1971)

Die Hauptangaben verdanke ich folgenden Herren: Buddhisten: Gebrüder Martinom (Tanjung)

Balinesische Hinduisten: Ana Agung Ketut Rai (Karangasem), Bayan Mangku, Bendesa Gedé Terna, Komang Nambi, Pemangku Gedé Karsa (alle in Tanjung), Ida Bagus Made Oka (Vorsteher des Agama Hindu für Lombok und Sumbawa, in Cakra Negara). Wetu Telu: Datoe Artadi (Tanjung), Datoe Soekawati (Mataram), Raden Sutasari (Bayan).

1) Abu Sufijan: Haupt des omajjadischen Klans im Stamme Qurais dem auch der Prophet angehörte, und einer der reichsten und einflußreichsten Männer der Handelsstadt Mekka.

Sein Sohn Mu'awija I, eine der hervorragendsten Herrscherfiguren der arabischen Geschichte, war der erste Kalif der Dynastie der 2) Omajjaden.

2) = Gläubiger; im Sinne: wer in seiner Seele und aufrichtig (innerlich) glaubt.

Für einen Sunniten ist jeder Rechtgläubige ein Mu'min. Die Gliederung in Muslim und Mu'min ist nach Ansicht der Sunniten eine willkürliche, ungerechtfertigte Trennung.

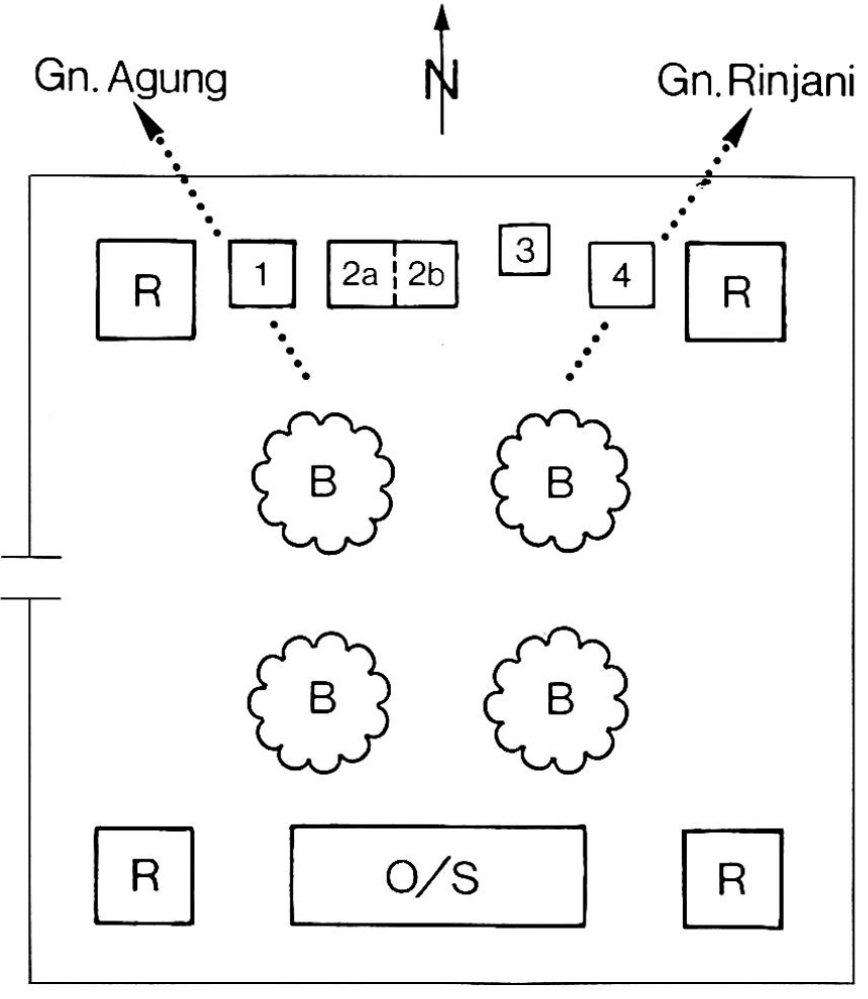

1: Schrein für Sang Hyang Tunggal: auf den Gottessitz in Bali, den Gunung Agung ( $3143 \mathrm{~m}$ ) ausgerichtet. Der Schrein liegt deshalb im Nordwesten des Gevierts und nicht im Nordosten, wie dies in den Tempeln Südbalis der Fall ist.

2a: Dem Mächtigen von Lombok, Gadoh, gewidmet.

2b: Sogenannter Bukit-Teil des Schreines. Der Name wird auf den BukitTempel (südöstlich von Karangasem) zurückgeführt. Er ist Bhatara Sakti Alit, dem Gottessohn der Königstochter Ayu Nyoman Winten, gewidmet.

Der Zusammenhang Lomboks und Balis kommt mit dieser Kombination unter einem Dach zum Ausdruck.

3: Ida Ngurah-Schrein (mit Steinen versehen). Alle dem Lingsar gewidmeten Tempelanlagen müssen auf Lombok Steingaben aufweisen.

4: Bhatara Gunung Rinjani gewidmeter Schrein, ausgerichtet auf den Gottessitz in Lombok, den Vulkan Rinjani (3775 m), deshalb im Nordosten des Gevierts gelegen.

R: Ruheplätze (Balé Piasan)

O/S: Platz für Opferbereitstellungen und Sitzplatz des Pedande (Brahmanenpriester)

B: Naga Sari-Bäume.

Das zweite Stockwerk ist Bhatara Lingsar gewidmet. Am 17. und 18. Dezember 1972 haben Wetu Telu, denen das Lingsar-Stockwerk, nicht aber der Gadoh, für Zeremonien zur Verfügung steht, über 100 Steine als Opfergaben gebracht. Die schwarzen, ungefähr $10 \mathrm{~kg}$ schweren Lavablöcke waren entweder in weiße Tücher mit weißen Bändern gewickelt oder mit gelben Tüchern (ohne Bänder) umhüllt. Man stellte sie sorgfältig ausgerichtet auf Gestelle. An der Wand dahinter hingen als Gaben von chinesischen Geschäftsleuten Spiegel mit Inschriften, die um Erfolg im Handel bitten. Bauern erflehen gesegnete Ernten mit Gaben an Dewi Sri oder mit Eierspenden für die weißen und schwarzen Aale im heiligen Teich, der Wischnu geweiht ist. 